\title{
Determination of Inspection Threshold Using Perceptive Sensitivities of Experienced Panel
}

\author{
Masao Nakagawa ${ }^{1}$, Hidetoshi Nakayasu ${ }^{2}$, and Tetsuya Miyoshi ${ }^{3}$ \\ ${ }^{1}$ Shiga University, Faculty of Economics, Center for Information Processing, \\ 1-1-1 Bamba, Hikone, Shiga 522-8522, Japan \\ ${ }^{2}$ Konan University, Faculty of Intelligence and Informatics, \\ 8-9-1 Okamoto, Higashinada, Kobe 658-8501, Japan \\ ${ }^{3}$ Toyohashi Sozo University, Faculty of Business and Informatics, \\ 20-1 Matushita, Ushikawacho, Toyohashi-shi, Aichi 440-8511, Japan \\ mnaka@biwako.shiga-u.ac.jp, nakayasu@konan-u.ac.jp, \\ miyoshi@sozo.ac.jp
}

\begin{abstract}
A determination of the threshold of inspection has been proposed based on the sensitivities by perception of panel. As the simulation in the proposed methods based on theory of signal detection are applied to determine the threshold level of experienced panels. It was found from the results that the value of index that evaluated the degree of experiensed skill enabled one to determine the threshold level of inspection.
\end{abstract}

Keywords: Theory of signal detection, Experienced panel, Stimulus threshold, Psychometric function.

\section{Introduction}

In the practical situation of quality control on visual inspection, a panel must decide the judgment of the quality by using one's sensitivities for products whether the quality of object is good or not. On the other hand, it is required in the field of automated visual inspection system with knowledge management to measure the characteristics of sensitivities for products and to evaluate the detective probability when the experienced skills were used for the judgment of inspection. In this case, it is an important viewpoint to obtain the skill of the professional sense.

The psychometric function method has been researched by the authors [3], though the classical methods of constant stimuli, such as limit and adjustment methods are applied until now [1]. A sorting performance by human vision is very flexible; therefore such a soft information processing has been regarded unfit on the computer which is good at digital information processing. The outline of the objectives in this study is drawn in Fig. 1. The approaches for knowledge management were illustrated in Fig. 1. The former management is two kind of stimulus as a mixture of signal and noise. On the other hand, the latter is concerned with the two kind of human response by knowledge and skill of experienced panel. However, it is hardly so far that defect inspection process using two kind of stimulus. To obtain the psychometric function 
for detecting the deference on the modalities of graphics, a prototype research was developed based on the constant stimulation method, has been proposed by the authors $[2,3,4]$.

The aim of this work is to discuss the potentiality of identifying human perception and skill of experienced panel, it is a new approach. In this paper, the simulation in the proposed methods based on theory of signal detection are applied to determine the level of threshold whether the quality of industrial object is good or not by perception performance of experienced panel. The computer simulation was performed to determine the thresolds for inspection.

\section{Measurement of the Degree of Skills}

\subsection{Strength of Stimulus and the Human Response}

The psychophysical method advanced by authors enables one to measure the perceptual skill related to the defect inspection. It seems to be possible to add the knowledge and the experience by requesting information obtained from the visual performance of the experienced panel. It is assumed in this paper that the difference of the figure attribute is detected in the defect detection work as shown in Fig. 1. As for this method, the discrimination capacity of man who inspects it by the paired comparison is based on the method of constant stimuli. Therefore, it is assumed in this paper that the human response is identified by the mathematical function of the strength of stimulus.
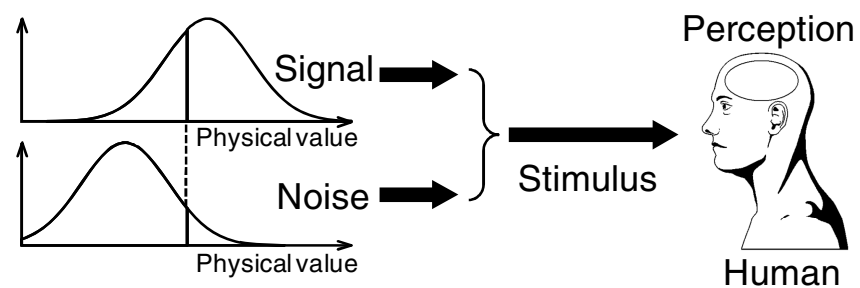

Human
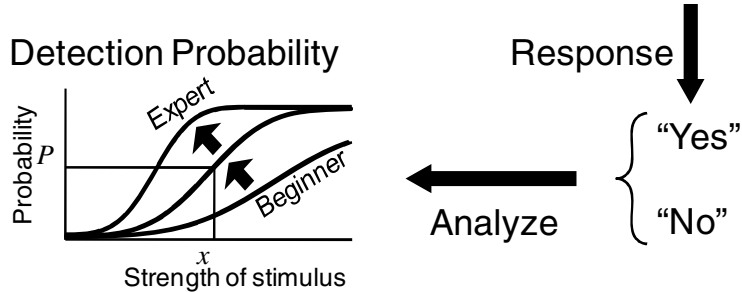

Fig. 1. Outline of concept

\subsection{Theory of Signal Detection}

As mentioned in 2.1, human response is identified by the mathematical function of the strength of stimulus. From this point of view, the model of human response is formulated by the theory of signal detection. In this formulation, there are two kinds 
of response and stimulus. The former consists of "yes" and "no", the latter consists of "signal" and "noise" as shown in Table 1. Even if the detection subject is a machine or human, the signal is detected from among the noise that always exists in the background. The level of the noise in the background is assumed to be random, it is generated from the outside or inside of the detection subject.

Table 1. Response of human by stimulus

\begin{tabular}{|c|c|c|c|}
\hline & \multicolumn{2}{|c|}{ Response of Human } \\
\hline & & Yes $(S N)$ & $\operatorname{No}(N)$ \\
\hline \multirow{2}{*}{ 氡 } & \begin{tabular}{|c|} 
Signal \\
+ \\
Noise \\
$(s n)$ \\
\end{tabular} & $\begin{array}{c}\text { A: hit } \\
P(S N \mid s n) \\
\text { OK }\end{array}$ & $\begin{array}{l}\mathrm{C}: \text { miss } \\
P(N \mid s n) \\
\quad \text { NG }\end{array}$ \\
\hline & $\begin{array}{c}\text { Noise } \\
(n)\end{array}$ & $\begin{array}{c}\text { B: false-alarm } \\
P(S N \mid n) \\
\text { NG }\end{array}$ & $\begin{array}{c}\text { D:correct-rejection } \\
P(N \mid n) \\
\text { OK }\end{array}$ \\
\hline
\end{tabular}

Table 1 shows the combination of the responses such as yes and no to the stimulus such as signal and the noise. The response to the stimulation of the SN distribution is said, "hit" for "yes" and "miss" for "no". When each probability is $P(S N \mid s n)$ and $P(N \mid s n)$ the relationship is as follow:

$$
P(S N \mid s n)+P(N \mid s n)=1
$$

If SN shows "signal-plus-noise", N shows "noise", the response to the stimulation N (noise) is called "false-alarm" when the response is "yes" and "correct-rejection" when the response is "no". If each probability is $P(S N \mid n)$ and $P(N \mid n)$, the relationship is

$$
P(S N \mid n)+P(N \mid n)=1
$$

Therefore, it is enough only by the calculation of $P(S N \mid s n)$ of "hit" and $P(S N \mid n)$ of "false-alarm".

The participant first receives stimulation, and the judgment from perception. They should judge whether stimulation is a signal or a noise in each trial. However, the judgment is difficult. One of the reasons is a random change of the noise that it strengthens and weakens. This situation can be shown in the Fig. 2 as two probability distributions that show a random change of the noise $(\mathrm{N})$ and the signal-plus-noise (SN) distributions. As for the perception, the $\mathrm{SN}$ distribution is always larger than $\mathrm{N}$ distribution, because the signal is added to the noise. When the strength of the signal weakens, the difference of the mean value of two distributions becomes small. If SN distribution corresponds to $\mathrm{N}$ distribution then the judgment is very difficult. A relative distance of the mean value of the $\mathrm{SN}$ distribution and $\mathrm{N}$ distribution becomes the index of the sensitivity of the detection that is called $d^{\prime}$. Let us designate by $\bar{x}_{s n}$, $\bar{x}_{n}$ the mean of the SN and $\mathrm{N}$ distributions. The distance $d^{\prime}$ is designated by the next equation.

$$
d^{\prime}=\bar{x}_{s n}-\bar{x}_{n}
$$

When $d^{\prime}$ is small, the detection by human becomes difficult. 


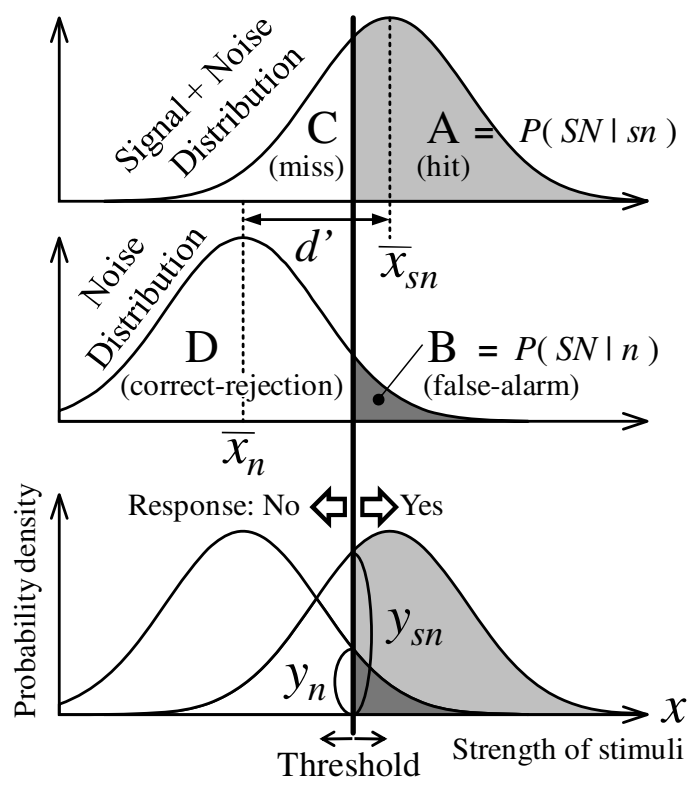

Fig. 2. The noise (N) and the signal-plus- noise (SN) distributions

The participant perceives when stimulation is strong or weaker than the standard values by method of constant stimuli, and responds with "Yes" or "No". They should respond yes-no for the stimulation that mixed the signal and the noise was received at random. In the physical value $x$ of stimulation, the probability density of $\mathrm{N}$ distribution is defined $y_{n}(x)$, the probability density of $\mathrm{SN}$ distribution is defined $y_{s n}(x)$. The ratio $l(x)$ is shown by the next equation.

$$
l(x)=\frac{y_{s n}(x)}{y_{n}(x)}
$$

This $l(x)$ is called a likelihood ratio. In the case where $l(x)$ is greater than 1.0 , the probability of responding for the $\mathrm{SN}$ distribution is larger than that of $\mathrm{N}$ [1].

\subsection{Measurement and Analysis}

It is known that in the event of the natural world, there is something that takes the distribution of the amount according to normal distribution because of central limit theorem. If one assumed that $\mathrm{N}$ distribution and $\mathrm{SN}$ distribution are among according to normal distribution, these probability density function are given by eq. (5).

$$
\begin{gathered}
y_{s n}(x)=\frac{1}{\sqrt{2 \pi} \sigma_{s n}} \exp \left[-\frac{1}{2}\left(\frac{x-\mu_{s n}}{\sigma_{s n}}\right)^{2}\right], \\
y_{n}(x)=\frac{1}{\sqrt{2 \pi}} \exp \left[-\frac{1}{2} x^{2}\right]
\end{gathered}
$$


The likelihood ratio is the following.

$$
l(x)=\frac{1}{\sigma_{s n}} \exp \left[-\frac{1}{2}\left(\frac{x-\mu_{s n}}{\sigma_{s n}}\right)^{2}+\frac{1}{2} x^{2}\right]
$$

It becomes the following when assuming that the variance of $\mathrm{N}$ and $\mathrm{SN}$ distribution is equal.

$$
l(x)=\exp \left[x \mu_{s n}-\frac{1}{2} \mu_{s n}^{2}\right]
$$

This is monotonically increasing function. Therefore the $l(x)$ is in proportion to strength of stimulus by physical value $x$.

Applying the probability function to the above equations, it is found the following results:

1) It is possible to evaluate the relationship between the signal and noise.

2) $d^{\prime}$ and $l(x)$ is able to calculated.

3) The validation methodology is given which of a sensitivity or a threshold influences.

\section{Practical Application}

\subsection{Design of Simulation for Sensory Tests}

The decision method of the inspection threshold is examined by applying the theory of signal detection about the relation between the judgment of the inspection panel and the received stimuli by signal of physical value. It assumes that it is stronger than the threshold, when the human is received the stimulation of SN and $\mathrm{N}$ distribution, and judges the defect.

In the case where the inspection panel judges the defect, there is probability $P(S N \mid s n)$ that correctly judges in the SN distribution, and is probability $P(S N \mid n)$ that makes a mistake in the $\mathrm{N}$ distribution. When you apply the signal detection theory to the design of the inspection standard value, it is necessary to enlarge $P(S N \mid s n)$ and to reduce $P(S N \mid n)$. It is suggested in the paper to calculate the best inspection threshold from the relation of this trade-off. Fig. 3 is the flowchart of the inspection threshold decision.

\subsection{Results and Discussions}

It is shown in Fig. 4 that the relation between the likelihood ratio by eq. (6) and the strength of stimulus $x$ is dependent on the ratio of $\bar{x}_{n} / \bar{x}_{s n}$. It is seen from the Fig. 4 the tendency of graph shows monotonically increasing function. However, it is also found that the likelihood ratio does not increases when the ratio of $\bar{x}_{n} / \bar{x}_{s n}$ becomes to be close to 1.0 . 


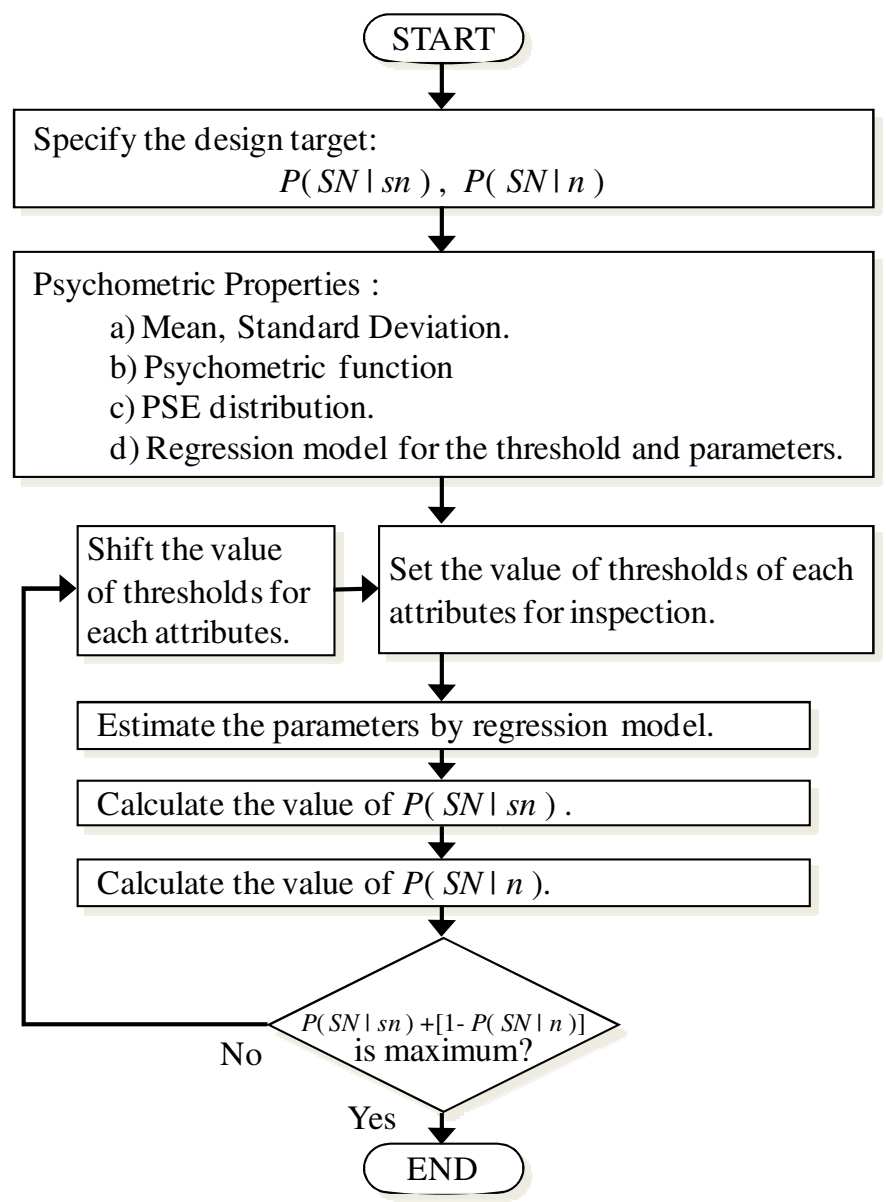

Fig. 3. Flowchart to determine the threshold in inspection

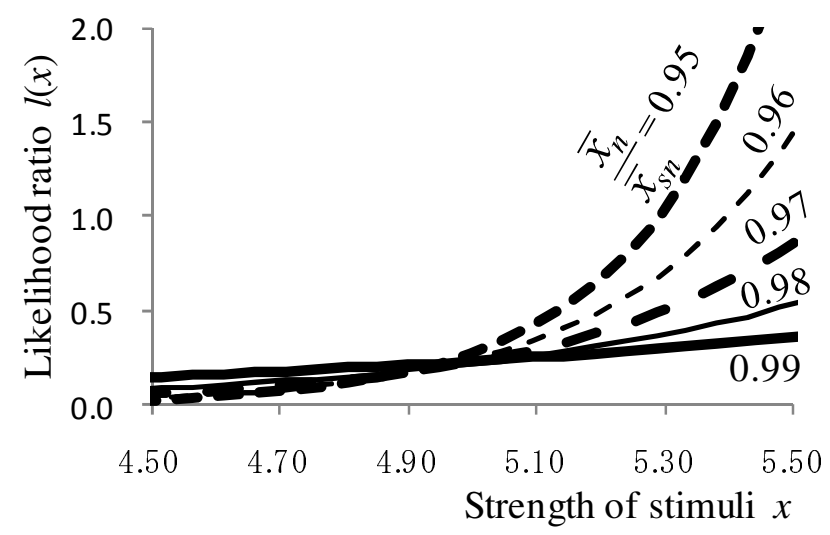

Fig. 4. Likelihood ratio in each $\frac{\bar{x}_{n}}{\bar{x}_{s n}}$ 


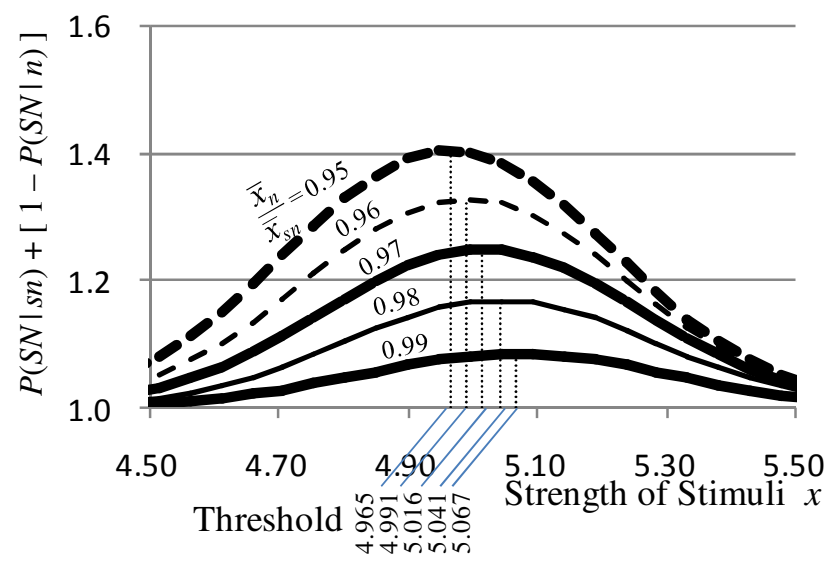

Fig. 5. Decided Threshold by $P(S N \mid s n)+[1-P(S N \mid n)]$ in each $\frac{\bar{x}_{n}}{\bar{x}_{s n}}$

Fig. 5 shows the summaries the algorithm of updating the new values of threshold level for visual inspection by iterating processes. These steps consists of

1) Experiment of paired comparisons

2) Analysis of psychometric function

3) Set the values of thresholds for each attributes and estimate the parameters of psychometric functions.

4) Estimate the parameter of psychometric functions for each attribute by regression model

5) Calculate $P(S N \mid s n)$ and $P(S N \mid n)$

6) If the value of $P(S N \mid s n)+[1-P(S N \mid n)]$ is not maximum, shift the value of threshold and go to step 3$)$. Otherwise, the process is end.

Fig. 5 shows that the threshold level is dependent on the ratio of $\bar{x}_{n} / \bar{x}_{s n}$ that is strongly correlated to the value of $d^{\prime}$.

Two main features in this study are as follows. The first is the likelihood ratio $l(x)$ means the judgment index of the human performance in visual perception, and the second is the distance $d^{\prime}$ between $\bar{x}_{s n}$ and $\bar{x}_{n}$ means the sensitivity of the human performance in visual perception,. This discussion will focus on the measurement for the skill level of experienced panel apply $l(x)$ and $d^{\prime}$.

The theory of signal detection can be applied to various kinds of fields. Because the sensitivity of the detection and the response to stimulation are individually appreciable, it was impossible in traditional psychophysics. It should be noted that the method of determining the level of threshold proposed in this paper is very efficient and improves the accuracy of the measurement of perceptive sensitivities of experienced panel for visual inspection. 


\section{Conclusion}

The following conclusions were derived from the results and discussions.

1) The judgment index clarified showing by the likelihood ratio.

2) It is found that $d^{\prime}$ means the sensitivity of the human performance in visual perception.

In the future it will be necessary to develop the experimental process for measure the likelihood $l(x)$ and sensitivities $d^{\prime}$ experienced panel in visual inspection.

Acknowledgments. This research was supported in part of IICT, Konan University by MEXT ORC JAPAN (2004-2008)

\section{References}

1. Ooyama, T., Imai, S., Wake, T. (eds.): Shinpen Kankaku Chikaku Handbook, Seishinshobo, pp. 19-41 (1994) (in Japanese)

2. Hayashi, H., Nakagawa, M., Nakayasu, H.: Psychometric evaluation with correlation for multiple perceptions. In: Proceedings of the First World Congress of the International Federation for Systems Research, CD-ROM, pp.S3-5-2. JAIST Press (2005)

3. Nakagawa, M., Nakayasu, H.: Evaluation of detection probability for flaw by multiple modalities. Journal of the Society of Materials Science 54(1), 2-7 (2005) (in Japanese)

4. Hayashi, H., Nakagawa, M., Nakayasu, H.: An evaluation method of human property using psychometric function in sensory test. Journal of the Chinese Institute of Industrial Engineers 24(4), 259-267 (2007) 